\title{
Desenvolvimento de pesquisas no Nordeste e a importância das Fundações de Amparo (2014-2016)
}

\section{Research development in the Northeast and the importance of Amparo Foundations (2014-2016)}

\author{
Vera Núbia Santos ${ }^{a}$ \\ (1D https://orcid.org/0000-0002-9040-7140 \\ Maria da Conceição Vasconcelos Gonçalves ${ }^{a}$ \\ (D) https://orcid.org/0000-0003-3902-3990 \\ Noêmia Lima Silva ${ }^{a}$ \\ (D) https://orcid.org/0000-0002-3845-445X
}

Resumo: A FAP é corresponsável pelo desenvolvimento científico, tecnológico e a inovação no país. Com o levantamento e análise de editais publicados no site das nove FAPs da região Nordeste, constatou-se o total de 316 editais entre 2014 e 2016 . Observa-se, em sua maioria, apoio para eventos, publicações e programas de pós-graduação, além de pesquisas. O Serviço Social deve atentar para a importância dessa agência de fomento, na perspectiva da qualidade da formação e do exercício profissional.

Palavras-chave: Ciência. Tecnologia e inovação. Fundação de Amparo à Pesquisa. Região Nordeste do Brasil. Serviço Social.

\begin{abstract}
FAP is co-responsible for scientific, technological development and innovation in the country. The survey and analysis of notices published on the website of the nine FAPs in the Northeast Region, found 316 notices between the years 2014 to 2016. It was found that the funding is mostly used for events, publications and postgraduate programs, in addition to research. Social Work must pay attention to the importance of this funding agency, in view of the quality of training and professional practice.
\end{abstract}

Keywords: Science. Technology and innovation. Research Support Foundation. Northeast Brazil. Social Work. 


\section{Introdução}

artigo advém do projeto "Pesquisa e pesquisadores em Serviço Social na região Nordeste: perfil, recursos e subsídios à formulação de políticas", cujo objetivo é analisar a produção de pesquisas financiadas por Fundações de Amparo à Pesquisa (FAPs) do Nordeste e o perfil de pesquisadoras(es) do Serviço Social e da Política Social. Os loci são as FAPs, responsáveis pelo fomento à pesquisa científica e tecnológica e pela inovação e, com isso, pelo incentivo ao desenvolvimento de políticas públicas na região. Tomando por base o Programa de Pós-Graduação em Serviço Social da Universidade Federal de Sergipe (Pross/ UFS), observa-se a contribuição da Fundação de Apoio à Pesquisa e à Inovação Tecnológica do Estado de Sergipe (Fapitec-SE) para consolidar a pós-graduação por meio de concessão de bolsas e com a publicação de doze coletâneas no período de 2015 a 2020.

O Serviço Social brasileiro alcançou, a partir de 1980, um legado intelectual intensificado pela pesquisa e produção do conhecimento, com a ampliação de programas de pós-graduação. A pesquisa, elemento fundamental da formação e do exercício profissional, contribui para o reconhecimento da profissão como área de conhecimento (Mota, 2013), o que a insere no campo das ciências sociais aplicadas nas agências de fomento à ciência, tecnologia e inovação (CT\&I), como o Conselho Nacional de Desenvolvimento Científico e Tecnológico (CNPq) e a Fundação Coordenação de Aperfeiçoamento de Pessoal de Nível Superior (Capes).

Há 36 programas de pós-graduação em Serviço Social no Brasil, e doze na região Nordeste. A pós-graduação está em universidades públicas, comunitárias e, em menor número, instituições privadas. No Nordeste, 100\% estão em universidades públicas: Ufma, Ufpi, Ufpe, UFRN e Ufal, com mestrado e doutorado; e Uern, Uece, UFPB, UEPB, UFS, Ufba e UFRB com mestrado.

O Pross/UFS, criado em 2011, está na décima turma, com 62 egressas(os), que, com as suas pesquisas, contribuíram na formulação de políticas e estratégias sociais para a população e inserem-se em espaços 
ocupacionais que têm nas políticas públicas a centralidade do exercício profissional.

A Avaliação Quadrienal de 2017 (Capes, 2017, p. 8) indica a área como um "lugar privilegiado de produção do conhecimento, dada a centralidade que a pesquisa científica neles assume [...em] temas como a questão social e as políticas sociais na contemporaneidade e o avanço teórico-metodológico do Serviço Social”, revelando um crescimento de quase $280 \%$ de cursos em dez anos, bem como da produção intelectual. A consolidação da área reflete aspectos da qualidade da produção e do perfil docente, mesmo com a maioria de programas em estratos médios de nota na Capes, também o perfil no Nordeste.

As agências de fomento têm papel preponderante na pesquisa desde a segunda metade do século XX. Nos estados, as FAPs induzem o desenvolvimento em CT\&I. As primeiras FAPs datam da década de 1960 e são disseminadas após a Constituição Federal (CF) de 1988, que trouxe dispositivos para facultar aos estados vinculação orçamentária para CT\&I. A CF expressa no Artigo 218 que “o Estado promoverá e incentivará o desenvolvimento científico, a pesquisa e a capacitação tecnológicas" (Brasil, 1988), o que se intensificou com a inclusão desse item nas Constituições estaduais.

No Nordeste, surgem a partir de 1989, chegando a todos os estados em 2003. De agência de fomento e difusão de pesquisa, que perscruta a realidade, com resultados que levem à implantação de políticas públicas, notou-se, entre 2014 e 2016, ao contrário, redução da indução à pesquisa, principalmente em políticas públicas, embora comuns editais direcionados à pós-graduação nos nove estados.

Embora se considere a crise política e econômica do Brasil no período, ressalta-se a ausência de um percentual orçamentário próprio para CT\&I. O país passou por momento conturbado de aumento das taxas de desemprego, da inflação, da diminuição do Produto Interno Bruto (PIB), em meio à reeleição da presidenta Dilma Rousseff, em 2014, e o seu impeachment, em agosto de 2016. 


\section{Breve apontamento: FAPs no Brasil e na região Nordeste}

As FAPs são uma das ações do Estado para o desenvolvimento da CT\&I. A primeira instituída no país foi a Fapesp, em 1962, ${ }^{1}$ dispondo de amparo em leis estaduais, exigência para a sua criação. A Fapesp é a principal em âmbito nacional e destaca-se como um organismo de apoio à pesquisa autônomo, eficiente e ágil nas decisões. Em 1964 surge no Rio Grande do Sul a Fapergs; em 1980, a Faperj; e a Fapemig, em 1985.

As FAPs têm a finalidade de administrar recursos e financiamento de pesquisas voltadas à CT\&I, bem como conceder bolsas para a capacitação de pesquisadoras(es) especialmente no âmbito das instituições de ensino superior. Estão submetidas às secretarias estaduais de Ciência e Tecnologia, que são responsáveis pelo repasse de recursos, e são articuladas pelo Conselho Nacional das Fundações de Amparo à Pesquisa (Confap). No incentivo à pesquisa consideram as características e interesses regionais e contam com o apoio do CNPq, da Capes e da Financiadora de Estudos e Projetos (Finep) em muitos convênios.

Chaves (2010) salienta a finalidade das FAPs no desenvolvimento de pesquisas para a formulação de políticas públicas, uma vez que têm por atribuição promover o desenvolvimento da pesquisa nas diversas áreas de conhecimento, da inovação tecnológica em estreita relação com o setor produtivo, do intercâmbio e divulgação científica, tecnológica e cultural, do estímulo à formação de recursos humanos, ao fortalecimento e à expansão da infraestrutura de pesquisas nos estados.

Há no país 26 FAPs, exceto em Roraima. No Nordeste, são: Fundação de Amparo à Ciência e Tecnologia do Estado de Pernambuco (Facepe), de 1989; Fundação de Amparo à Pesquisa do Estado de Alagoas (Fapeal), de 1990; Fundação Cearense de Apoio ao Desenvolvimento Científico e Tecnológico (Funcap), de 1990; Fundação de Apoio à Pesquisa do Estado

\footnotetext{
A Fundação de Amparo à Pesquisa do Estado de São Paulo (Fapesp) foi formalmente criada em 1960 (Lei Orgânica n 5.918, de 18 de outubro de 1960) e começou a funcionar efetivamente em 1962 (Decreto no 40.132, de 23 de maio de 1962).
} 
da Paraíba (Fapesq), de 1992; Fundação de Amparo à Pesquisa do Estado do Piauí (Fapepi), de 1993; Fapitec, de 1999;² Fundação de Amparo à Pesquisa do Estado da Bahia (Fapesb), de 2001; Fundação de Amparo à Pesquisa e ao Desenvolvimento Científico e Tecnológico do Maranhão (Fapema), de 2003; Fundação de Apoio à Pesquisa do Estado do Rio Grande do Norte (Fapern), de 2003.

A expansão das FAPs motivou a instituição da Lei n 13.243/2016 (Brasil, 2016a), mas a crise política agrava a economia e induz à implantação de um novo regime fiscal, mediante a Emenda Constitucional $n^{\circ}$ 95/2016 (Brasil, 2016b), que "congelou" os recursos federais por vinte anos e influenciou a redução de recursos para pesquisas. Extraiu-se dos editais das FAPs do Nordeste nos anos de 2014 a 2016 dados para compreender o apoio ao desenvolvimento da CT\&I na região, por meio do seu fomento.

\section{Percurso metodológico e alguns resultados}

Uma pesquisa direciona-se para a compreensão de determinado objeto num contexto da realidade e busca responder objetivamente à complexidade dos problemas sociais que afetam sujeitos concretos. Ela favorece as mediações entre os resultados e a dinâmica da realidade, fomenta e acompanha a implementação de políticas públicas, constituindo-se num instrumento de enfrentamento às históricas desigualdades sociais regionais.

A pesquisa, de natureza quanti-qualitativa, adotou o percurso metodológico da coleta sistemática de dados nos estados da região, em três grupos (por aproximação territorial): Bahia (BA), Sergipe (SE) e Alagoas (AL); Pernambuco (PE), Paraíba (PB) e Rio Grande do Norte (RN); e Maranhão (MA), Ceará (CE) e Piauí (PI). Utilizou-se o Programa Institucional de Bolsas de Iniciação Científica (Pibic), como caminho para articular

2 Criada em 1999 como Fundação de Amparo à Pesquisa de Sergipe (FAP-SE), em 2005 mudou a nomenclatura e ampliou os objetivos. 
discentes da graduação e da pós-graduação, amparados nas informações e documentos disponibilizados nos sites das FAPs. Inicialmente, fez-se a identificação das FAPs, com histórico e dados. Em seguida, fez-se levantamento de editais para obter as seguintes informações: recursos disponíveis; projetos aprovados, em especial de políticas públicas. Paralelamente, foi feito o levantamento documental das fontes primárias e secundárias, bem como pesquisa bibliográfica para alicerçar as análises.

A análise dos relatórios de IC coordenados pelas autoras e desenvolvidos por Gouveia (2019, 2020), que pesquisou as FAPs de PE, PB e RN, por Mota (2019) e Prudêncio (2020), responsáveis pelas FAPs de BA, AL, SE, e por Santos e Costa (2019) e Santos (2020), que pesquisaram as FAPs do MA, PI e CE, possibilitou a identificação de 316 editais nos sites das FAPs, como aponta o Quadro 1.

O total por ano revela a diminuição de editais e decorrente redução de recursos para as FAPs, em especial em 2016, ano do processo de impeachment da presidenta e da aprovação da EC n० 95. Os dados evidenciam a pouca expressividade de editais direcionados ao desenvolvimento das políticas públicas: constatam-se editais da Fapepi, em 2014, e da Fapema e da Funcap, em 2015. A Fapern, Fapesq, Fapitec e a Fapeal não lançaram editais com esse foco no período analisado.

É possível observar em todas as FAPs o apoio para organização/ participação em eventos científicos; publicação de trabalhos; concessão de bolsas de pós-graduação e IC, entre outros aspectos que denotam o foco na política de educação. Houve editais para contratação de pessoal via processo seletivo e para eleição de vaga no Conselho Superior da FAP. Deve-se salientar a existência de projetos de programas de pós-graduação em Serviço Social nos vários editais.

No período, a Fapema, com 67 editais, a Facepe, com sessenta, e a Fapesb, com 51, são as FAPs com mais difusão de recursos. Em todo o período houve a redução de editais (e de recursos), exceto na Fapesq, que passou de 132 para 86. Em FAPs com mais de vinte editais em 2014, por exemplo, houve redução significativa em 2016, à exceção da Facepe. 
No triênio, chama a atenção o reduzido número de editais na Fapesq e Fapern identificados nos sites.

Quadro 1. Número de editais por ano e por FAP

\begin{tabular}{|l|l|c|c|c|c|}
\hline UF & Sigla & $\mathbf{2 0 1 4}$ & $\mathbf{2 0 1 5}$ & $\mathbf{2 0 1 6}$ & Total \\
\hline MA & Fapema & 23 & 40 & 4 & $\mathbf{6 7}$ \\
\hline PI & Fapepi & 28 & 7 & 10 & $\mathbf{4 5}$ \\
\hline CE & Funcap & 8 & 14 & 6 & $\mathbf{2 8}$ \\
\hline Subtotal & & & & & $\mathbf{1 4 0}$ \\
\hline RN & Fapern & 8 & 3 & 4 & $\mathbf{1 5}$ \\
\hline PE & Facepe & 24 & 14 & 22 & $\mathbf{6 0}$ \\
\hline PB & Fapesq & 1 & - & 6 & $\mathbf{7}$ \\
\hline Subtotal & & & & & $\mathbf{8 2}$ \\
\hline AL & Fapeal & - & 8 & 15 & $\mathbf{2 3}$ \\
\hline SE & Fapitec & 12 & 1 & 7 & $\mathbf{2 0}$ \\
\hline BA & Fapesb & 28 & 11 & 12 & $\mathbf{5 1}$ \\
\hline Subtotal & & & & $\mathbf{9 6}$ & $\mathbf{3 1 6}$ \\
\hline Total & & $\mathbf{1 3 2}$ & $\mathbf{9 8}$ & & \\
\hline
\end{tabular}

Fonte: Elaborado pelas autoras a partir dos relatórios de iniciação científica.

Maranhão, Piauí e Ceará apresentaram o maior número de editais no período, destacando-se o ano 2015 como de maior concentração de editais na Facema e na Funcap. Dos 140 editais analisados, constatou-se que 21 referiam-se às políticas públicas. Desse conjunto, em 2014, só foram localizados três editais da Fapepi com esse foco. Na Fapema, quinze foram alusivos a projetos de pesquisa, sendo sete para políticas públicas. Ressalta-se, entre os editais de pesquisa, quatro para cursos de pós-graduação stricto sensu, um para estágio pós-doutoral, um para instituições reconhecidas pela Capes no Brasil e outro para programas de reconhecida excelência no exterior. Em relação à Funcap, dos catorze 
editais analisados em 2015, dois não eram projetos de pesquisa, mas houve onze para políticas públicas. Observou-se que cinco editais para a pós-graduação stricto sensu, sendo quatro referentes às políticas públicas (Santos e Costa, 2019). Na Fapepi, em 2015, não houve edital para políticas públicas. Dos projetos de pesquisa, três referiam-se a bolsas de pós-graduação.

Deve-se ressaltar que no Maranhão e no Piauí há programas de pós-graduação em políticas públicas, com mestrado e doutorado, o que pode estimular projetos de pesquisas. Na comparação com 2014, observa-se no Maranhão e no Ceará um aumento de editais de projetos de pesquisa, inclusive de políticas públicas. Já o Piauí reduziu editais entre 2014 e 2015, com um pequeno acréscimo em 2016. Em 2014, a Fapepi lançou três editais referentes às políticas públicas, o que não se verificou nos anos seguintes.

Dos 82 editais no Rio Grande do Norte, Paraíba e Pernambuco, apenas na Facepe há editais para políticas públicas: os dados revelaram em 2014 dez editais. A Fapesq teve como objeto processo seletivo para contratação de pessoal e a Fapern não teve edital com esse foco (Gouveia, 2019). Em 2015, a Facepe teve quatro editais voltados para bolsas de pesquisa, dois para apoio à cooperação científica, um para apoio de atividades de monitoria, um para eleição de membro do Conselho Superior, e seis para o estímulo, fortalecimento e apoio a atividades de pesquisa e produção crítica de conhecimento. A Fapern publicou três editais: dois para concessão de bolsas de pesquisa e um para apoio à realização de eventos C\&T. Na Fapesq não foram encontrados editais do ano de 2015. Em 2016, foram identificados seis editais da Fapesq, 22 da Facepe e quatro da Fapern, com conteúdos semelhantes aos anteriores. Deve-se ressaltar nesses estados os programas de pós-graduação em Serviço Social consolidados, com dois doutorados (Ufpe e UFRN), o que constitui um fator importante para as pesquisas.

Na Bahia, Sergipe e Alagoas foram 94 editais no período de análise. Em 2014, quarenta editais: 28 da Fapesb e doze da Fapitec. Em 2015, 
foram onze editais da Fapesb, oito da Fapeal e um da Fapitec, direcionado à concessão de bolsas de mestrado e doutorado (Mota, 2019), e tiveram como objetos a cooperação internacional, o apoio a projetos de pesquisa para jovens cientistas, as bolsas de mestrado, doutorado e de pós-doutorado, os projetos de pesquisa e produção de conhecimento na área de tecnologia da informação e comunicação, as propostas de apoio à organização de eventos científicos e/ou tecnológicos, o apoio a projetos de pesquisa para atração de pesquisador visitante estrangeiro, o programa de apoio às pesquisas em empresas, o apoio financeiro para investimento em infraestrutura de pesquisa e a pesquisadores de diversas áreas do conhecimento. No caso do Pross, no período analisado, sete discentes concluíram o mestrado com bolsas da Fapitec. Na Fapeal, houve chamada para composição das câmaras permanentes de avaliação e assessoramento. Em 2016, foram 34 editais, nenhum direcionado às políticas públicas, com focos semelhantes aos anteriores. Nesses estados há quatro programas de pós-graduação em Serviço Social, um com doutorado (Ufal), que podem suscitar o financiamento para pesquisas.

As FAPs do Nordeste apresentam, em síntese, um espelho da crise que atinge a realidade brasileira, com redução abrupta do fomento à pesquisa e à produção de conhecimento em CT\&I, devido à diminuição de recursos orçamentários, o que impede, inclusive, o fomento à pesquisa sobre políticas públicas.

A conjuntura brasileira, que em momento de pandemia do coronavírus ainda se calca em um governo de extrema-direita, conservador na política e liberal na economia, aprofunda a fragmentação do que historicamente se constrói em CT\&I no país. A escassez de recursos acentua a frágil indução às pesquisas sobre políticas públicas, uma vez que o minimalismo do Estado se exacerba, excluindo do patamar de direitos o acesso a políticas que minimizem as desigualdades do país.

Para o Serviço Social, essa redução de fomento às pesquisas traz implicações que rebatem nas competências e atribuições privativas da profissão, como preveem os artigos $4^{\circ}$ e $5^{\circ}$ da Lei no 8.662 (Brasil, 1993). 
Ao pensar as FAPs como instâncias que estimulam a CT\&I, deve-se ter no horizonte o seu papel para as políticas públicas e, nesse sentido, a profissão deve fazer parte desse caminho.

\section{Considerações finais}

O fortalecimento das FAPs nos anos 1990 previa a instituição de benefícios à população a longo prazo, por meio da indução ao desenvolvimento da CT\&I. Ante uma conjuntura econômica limitadora de recursos para essa finalidade, essa previsão perde o sentido. $O$ corte de verbas no orçamento da CT\&I, que se intensificou a partir de 2013 e chega ao ápice de restrição com a Emenda constitucional no 95/2016, ${ }^{3}$ interfere no fomento à pesquisa e, no caso das FAPs, representa uma ameaça à sociedade, visto que limita pesquisas nas políticas públicas que poderia influir na análise das desigualdades sociais.

Atualmente há pouco incentivo nas FAPs do Nordeste à pesquisa. Compreende-se a importância das agências no avanço do conhecimento, porém se percebe o distanciamento no que tange às políticas públicas nos últimos anos, no contexto institucional, em questões importantes tanto para o Estado quanto para a sociedade. Apesar do contexto adverso, o Pross ampliou a produção científica de docentes e discentes com aprovação dos editais Capes/Fapitec/SE ns 09/2013 e 11/2016, que possibilitou a publicação de doze coletâneas.

A pesquisa traz contribuições para repensar o Estado, o fomento à pesquisa e os seus rebatimentos na sociedade, além de propiciar a contribuição da área. A participação de pesquisadoras(es) tende a aumentar caso o financiamento público à pesquisa torne-se uma política no país,

\footnotetext{
Dados disponíveis no site https://auditoriacidada.org.br/categoria-conteudo/graficos/ indicam redução da execução orçamentária em ciência e tecnologia em nível federal: em 2013, a área foi responsável por 0,38\% dos recursos executados, chegando em 2019 a 0,23\%. Nos anos de 2014, 2015 e 2016, foram executados, respectivamente, $0,28 \%, 0,27 \%$ e $0,24 \%$.
} 
como no caso de fomento pelo CNPq, que, ainda que reduzidos, mantém os principais editais. Há que se recuperar o papel das FAPs, articulado aos programas de pós-graduação da área, para consolidar e ampliar a difusão de conhecimentos.

\section{Referências}

BRASIL. Constituição da República Federativa do Brasil. Brasília: Presidência da República, Casa Civil, 1988.

BRASIL. Lei n 8.662, de 7 de junho de 1993. Dispõe sobre a profissão de assistente social e dá outras providências. Brasília: Presidência da República, Casa Civil, 1993.

BRASIL. Lei no 13.243, de 11 de janeiro de 2016. Dispõe sobre estímulos ao desenvolvimento científico, à pesquisa, à capacitação científica e tecnológica e à inovação e altera a Lei $n^{\circ} 10.973$, de 2 de dezembro de 2004, a Lei $n^{\circ} 6.815$, de 19 de agosto de 1980, a Lei $n^{\circ}$ 8.666, de 21 de junho de 1993, a Lei no 12.462, de 4 de agosto de 2011, a Lei no 8.745 , de 9 de dezembro de 1993, a Lei no 8.958, de 20 de dezembro de 1994, a Lei no 8.010, de 29 de março de 1990, a Lei no 8.032, de 12 de abril de 1990, e a Lei no 12.772, de 28 de dezembro de 2012, nos termos da Emenda Constitucional no 85, de 26 de fevereiro de 2015. Diário Oficial da União, Brasília, 12 dez. de 2016a. Disponível em: http://www. planalto.gov.br/ccivil_03/_ato2015-2018/2016/lei/113243.htm. Acesso em: 11 mar. 2020.

BRASIL. Emenda Constitucional no 95, de 15 de dezembro de 2016. Altera o ato das disposições constitucionais transitórias para instituir o novo regime fiscal e dá outras providências, $2016 \mathrm{~b}$.

CAPES. Relatório de Avaliação Quadrienal 2017 Serviço Social. Brasília: Ministério da Educação, Coordenação de Aperfeiçoamento de Pessoal de Nível Superior, Diretoria de Avaliação, 2017.

CHAVES, V. L. J. Fundações de Amparo à Pesquisa. In: OLIVEIRA, D. A.; DUARTE, A. M. C.; VIEIRA, L. M. F. Dicionário: trabalho, profissão e condição docente. Belo Horizonte: UFMG/Faculdade de Educação, 2010. 1 CD-ROM.

GOUVEIA, D. dos S. Fundações públicas, pesquisa e pesquisadores de Serviço Social em 2015 (PE, PB, RN). Relatório final de iniciação científica. Programa Institucional de Bolsas de Iniciação Científica, Universidade Federal de Sergipe, São Cristóvão, 2019.

GOUVEIA, D. dos S. Fundações públicas, pesquisa e pesquisadores de Serviço Social em 2015 (PE, PB, RN). Relatório parcial de iniciação científica. Programa Institucional de Bolsas de Iniciação Científica, Universidade Federal de Sergipe, São Cristóvão, 2020. 
MOTA, A. E. Serviço Social brasileiro: profissão e área de conhecimento. Revista Katálysis, Florianópolis, v. 16, n. esp., p. 17-27, 2013.

MOTA, R. L. S. da. Fundações públicas, pesquisa e pesquisadores de Serviço Social em 2015 (BA, SE, $A L)$. Relatório Final de Iniciação Científica. Programa Institucional de Bolsas de Iniciação Científica, Universidade Federal de Sergipe, São Cristóvão, 2019.

PRUDÊNCIO, W. T. L. Pesquisa e pesquisadores em Serviço Social no Nordeste: fundações públicas em BA, SE e AL (2016). Relatório parcial de Iniciação Científica. Programa Institucional de Bolsas de Iniciação Científica, Universidade Federal de Sergipe, São Cristóvão, 2020.

SANTOS, K. P. dos. Pesquisa e pesquisadores em Serviço Social no Nordeste: fundações públicas em MA, PI e CE (2016). Relatório parcial de iniciação científica. Programa Institucional de Bolsas de Iniciação Científica, Universidade Federal de Sergipe, São Cristóvão, 2020.

SANTOS, M. S. E.; COSTA, L. O. Pesquisa e pesquisadores em Serviço Social no Nordeste: fundações públicas em MA, PI e CE (2015). Relatório final de iniciação científica. Programa Institucional de Bolsas de Iniciação Científica, Universidade Federal de Sergipe, São Cristóvão, 2019.

\section{Sobre as autoras}

Vera Núbia Santos - Doutora em Serviço Social, Universidade Federal de Sergipe (UFS), departamento e pós-graduação em Serviço Social.

E-mail:venus_se@uol.com.br

Maria da Conceição Vasconcelos Gonçalves - Doutora em Serviço Social, Universidade Federal de Sergipe (UFS), pós-graduação em Serviço Social.

E-mail: licavasconcelos@gmail.com

NoÊmia Lima SiLVA - Doutora em Educação, Universidade Federal de Sergipe (UFS), departamento e pós-graduação em Serviço Social.

E-mail:sany25@uol.com.br 